\title{
Bestimmung von Organdosen und effektiven Dosen in der Radioonkologie
}

\author{
Jakob Roth, Aurélien Emmanuel Martinez ${ }^{1}$
}

\begin{abstract}
Hintergrund und Ziel: Mit zunehmender Heilungschance in der Radioonkologie muss auch das durch Streustrahlung induzierte Strahlenrisiko außerhalb des Zielvolumens beurteilt werden können. Das Krebsrisiko einer Strahlenbehandlung kann aufgrund von Organdosen und einer etwas eingeschränkten effektiven Dosis abgeschätzt und verglichen werden.

Material und Methodik: Die Dosen in strahlenempfindlichen Organen außerhalb der Strahlenfelder können mit Hilfe des PC-Programms PERIDOSE von van der Giessen abgeschätzt werden. Die effektiven Dosen werden nach dem Konzept der ICRP bestimmt, wobei jedoch das Zielvolumen und die damit zusammenhängenden Organe unberücksichtigt bleiben.

Ergebnisse: Organdosen außerhalb der Strahlenfelder sind meist < $1 \%$ der Dosis im Zielvolumen, in Einzelfällen jedoch bis zu etwa 3\%. Die effektiven Dosen während einer Strahlentherapie betragen je nach Zielvolumen, angewendeter Bestrahlungstechnik und applizierter Dosis im ICRU-Punkt zwischen 60 und 900 mSv.

Schlussfolgerung: Für die Abschätzung von Strahlenrisiken können Organdosen in der Radioonkologie mit Hilfe des Programms PERIDOSE berechnet werden. Für die Bewertung des Strahlenrisikos nach ICRP muss bei der effektiven Dosis das oft fortgeschrittene Alter der Patienten berücksichtigt werden, damit z.B. die hohen Gonadendosen für die effektive Dosis nicht überbewertet werden.
\end{abstract}

Schlüsselwörter: Organdosen · Effektive Dosis · Strahlenrisiko · Hochvolttherapie

Strahlenther Onkol 2007;183:392-7

DOI 10.1007/s00066-007-1697-y

Determination of Organ Doses and Effective Doses in Radiooncology

Background and Purpose: With an increasing chance of success in radiooncology, it is necessary to estimate the risk from radiation scatter to areas outside the target volume. The cancer risk from a radiation treatment can be estimated from the organ doses, allowing a somewhat limited effective dose to be estimated and compared.

Material and Methods: The doses of the radiation-sensitive organs outside the target volume can be estimated with the aid of the PC program PERIDOSE developed by van der Giessen. The effective doses are determined according to the concept of ICRP, whereby the target volume and the associated organs related to it are not taken into consideration.

Results: Organ doses outside the target volume are generally $<1 \%$ of the dose in the target volume. In some cases, however, they can be as high as $3 \%$. The effective doses during radiotherapy are between 60 and $900 \mathrm{mSv}$, depending upon the specific target volume, the applied treatment technique, and the given dose in the ICRU point.

Conclusion: For the estimation of the radiation risk, organ doses in radiooncology can be calculated with the aid of the PC program PERIDOSE. While evaluating the radiation risk after ICRP, for the calculation of the effective dose, the advanced age of many patients has to be considered to prevent that, e.g., the high gonad doses do not overestimate the effective dose.

Key Words: Organ doses · Effective dose · Radiation risk · Megavoltage therapy

\section{Einleitung}

Die effektive Dosis wurde von der ICRP [7] definiert, um ein Gesamtrisiko von Strahlenexpositionen anzugeben. Zur Bestimmung dieser Größe ist eine umfassende Kenntnis der Dosisverteilung im gesamten Körper erforderlich. In der diagnostischen Radiologie wird die Strahlenexposition bei einer
Untersuchung üblicherweise in Form der effektiven Dosis angegeben. Diese kann direkt in Beziehung gesetzt werden zum Strahlenkrebsrisiko. Dieses wird für den strahleninduzierten Krebstodesfall mit 5\% pro Sv angegeben [7].

Die effektive Dosis bezieht sich nur auf stochastische Effekte (Dosis < $1 \mathrm{~Sv}$ ). In der Radioonkologie treten jedoch

\footnotetext{
${ }^{1}$ Radiologische Physik, Radio-Onkologie, Universitätsspital, Basel, Schweiz.
} 
insbesondere im Zielvolumen Dosen im deterministischen Bereich auf ( $>1 \mathrm{~Sv}$ ). Für Risikoorgane außerhalb des Zielvolumens und außerhalb der Strahlenfelder liegen die Dosen im Bereich der stochastischen Effekte. Somit kann mit diesen Organdosen eine etwas abgeänderte effektive Dosis für die Strahlentherapie und damit auch das betreffende Strahlenrisiko bestimmt werden [9]. Das Zielvolumen bzw. die darin applizierte Dosis wird dabei nicht berücksichtigt. Begründet werden kann dieses Vorgehen damit, dass das Zielvolumen kein Risikoorgan darstellt, sondern darin ein Strahleneffekt erzielt werden soll.

Insbesondere bei kurativer Strahlenbehandlung, bei jüngeren Patienten oder bei späterem Kinderwunsch ist in der Radioonkologie ein potentielles Strahlenrisiko zu berücksichtigen. Das Risiko einer Krebsinduktion bzw. eines Sekundärmalignoms gewinnt mit zunehmender Heilungschance und Überlebenszeit des Patienten nach einer Strahlentherapie an Bedeutung [5].

Auch die UNSCEAR stellte in ihren letzten Reporten $[12,13]$ Überlegungen zu dieser Fragestellung an. In ihrem Bericht des Jahres 2000 [13] weist die Kommission darauf hin, dass die Größe der effektiven Dosis zwar ungeeignet für die Charakterisierung therapeutischer Bestrahlungen sei, weil deterministische Effekte im Zielvolumen beabsichtigt seien. Sie empfiehlt jedoch, effektive Dosen aus der Streustrahlung außerhalb des Zielvolumens zu bestimmen. Daraus könnten Kollektivdosen für die Strahlentherapie berechnet werden. Hinweise für Risikoschätzungen werden bei erfolgreicher Heilung durch Strahlentherapie als besonders wichtig beurteilt.

Die Dosis außerhalb des Nutzstrahlenfeldes setzt sich aus mehreren Strahlungsbeiträgen zusammen. Für die Bestimmung einer Organdosis muss zwischen den folgenden Herkunftsorten unterschieden werden:

- externe Streustrahlung vom Strahlerkopf: Durchlass- und Streustrahlung (Blenden, Keilfilter, Abschirmblöcke, Trägerplatte usw.),

- externe Streustrahlung aus dem Bestrahlungsraum (z.B. Tisch, Wände),

- interne Streustrahlung vom Patienten.

Für die Bestimmung von Organdosen außerhalb des Zielvolumens stehen verschiedene Möglichkeiten zur Verfügung. Die genaueste, aber aufwendigste Methode ist die Messung am anthropomorphen Phantom bei der Simulation von Bestrahlungen [4]. Mit tabellierten Werten von Durchlass- und Streustrahlung aus dem Strahlerkopf und vom Bestrahlungsraum sowie Streustrahlung vom Patienten kann die Dosis in Organen außerhalb des Nutzstrahls abgeschätzt werden $[9,15]$. Diese Methode wurde mit dem PC-Programm PERIDOSE von van der Giessen verfeinert und erlaubt eine einfache Dosisbestimmung an Punkten außerhalb des Zielvolumens aufgrund der wichtigsten Bestrahlungsparameter eines Feldes [14]. Dafür wenig geeignet sind hingegen die kommerziellen Bestrahlungsplanungssysteme, da damit die relativ kleinen Dosen teilweise stark unterschätzt werden. Der Algorithmus ist grundsätzlich nicht für relative Dosen $<3 \%$ der Zielvolumendosis ausgelegt.

\section{Material und Methodik}

Die betreffenden Organdosen wurden mit dem PC-Programm PERIDOSE (Version 1.10) berechnet, welches van der Giessen erstellt und zur Verfügung gestellt hat [14]. Sie sind abhängig von verschiedenen Größen, insbesondere vom Abstand zwischen Zielvolumen und Organ, von der Feldgröße und von der Photonenenergie. Die erforderlichen Distanzangaben vom Patienten sind in Abbildung 1 dargestellt. Die folgenden Bestrahlungsparameter werden berücksichtigt: Strahlenenergie, Einstrahlrichtung, Tiefe des Isozentrums, Feldgröße (im Isozentrum) unter Berücksichtigung einer irregulären Form, Abschirmblöcke und Trägerplatte, Keilfilter, Schwächung bei Durchstrahlung des Tisches.

Bei den Bestrahlungen der einzelnen Zielvolumina mussten bis zu vier Serien berücksichtigt werden, die sich z.B. in den Feldgrößen bzw. -formen unterschieden (Anpassungen an das Zielvolumen). Auch ein allfälliger Boost mit Photonenstrahlung wurde in die Berechnung miteinbezogen. Der Beitrag jedes einzelnen Feldes bei der Bestrahlung eines Zielvolumens muss unter Berücksichtigung der entsprechenden Parameter bestimmt werden.

Im PERIDOSE-Programm kann ein $60^{\circ}$-Keil verwendet werden. Wie in der Praxis lassen sich kleinere Keilfilterwinkel durch Kombination von offenem Feld und Keilfilterfeld realisieren. Es kann unterschieden werden zwischen senkrecht auf

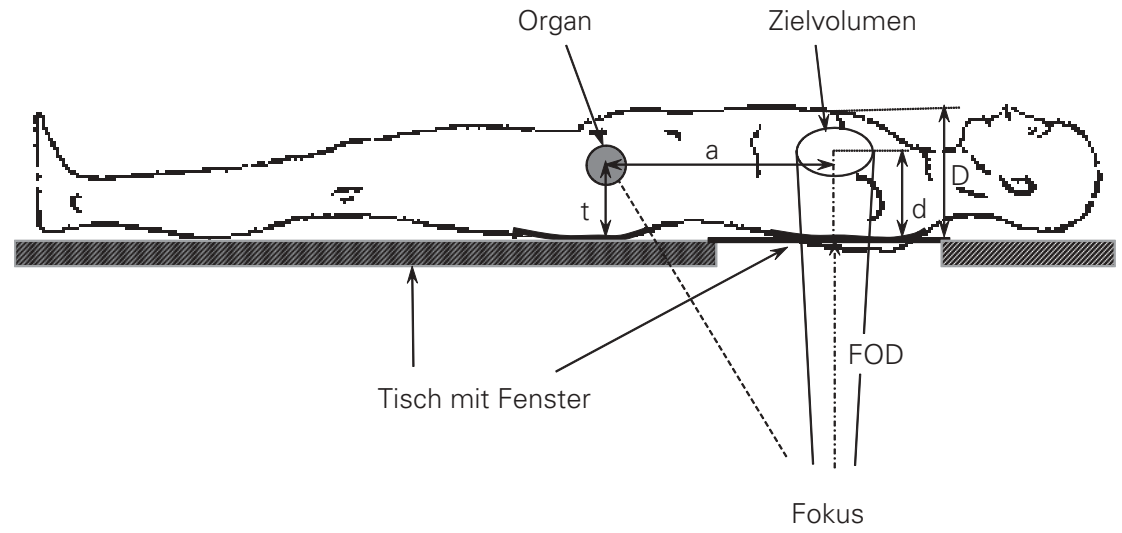

Abbildung 1. Bezeichnung der geometrischen Parameter bei der Bestimmung der Organdosis. a: Distanz zwischen Feldmitte und Organmittelpunkt; D: Durchmesser des Patienten im Zentralstrahl; d: Tiefe des Isozentrums; FOD: Fokus-Oberflächen-Distanz; t: Tiefe des Organs in Strahlrichtung.

Figure 1. Designation of the geometric parameters for the determination of the organ dose. a: distance of the organ's center from the field center; D: patient's diameter in the beam axis; $\mathrm{d}$ : depth of the isocenter; FOD: focus-surface distance; $t$ : depth of the organ in beam direction. 
die Körperoberfläche auftreffendem Zentralstrahl, IMRT (intensitätsmodulierte Radiotherapie) und tangentialer Bestrahlung, wie z.B. bei der Mammabestrahlung.

Die effektive Dosis berechnet sich als Summe von Organdosen, die mit Risikofaktoren gewichtet sind. Das Zielvolumen und direkt angrenzende Organe werden in dieser Arbeit nicht berücksichtigt. Zu den Risikoorganen mit vorgegebenen Wichtungsfaktoren $\mathrm{w}_{\mathrm{T}}$ gehören gemäß ICRP [7]: Gonaden $(0,20)$, Lungen, Magen, Dickdarm, rotes Knochenmark (je 0,12), Schilddrüse, Speiseröhre, Mamma, Leber, Blase (je 0,05), Haut und Knochenoberfläche (je 0,01). Zusätzlich zu diesen Risikoorganen müssen noch fünf weitere Organe mit den höchsten Dosen erfasst werden, als Beitrag zum Restkörper (Remainder, $\left.\mathrm{w}_{\mathrm{T}}=5 \times 0,01\right)$. Bei größeren Organen, wie z.B. der Lunge, wird die Dosis in mehreren Punkten bestimmt und ein Mittelwert gebildet. Schwierig ist vor allem die Bestimmung der mittleren Dosen von rotem Knochenmark, Haut und Knochenoberfläche. Für diese Organsysteme wurde ein gewichteter Durchschnittswert aus Dosen an verschiedenen Körperstellen bestimmt, unter Berücksichtigung der betreffenden Gewebeanteile. Die Hautdosen wurden an der Oberfläche eines Alderson-Phantoms mit Halbleiterdioden gemessen. Die Dosen für das rote Knochenmark und die Knochenoberfläche wurden als gleich angenommen. Bei der Mammabestrahlung wurde die mittlere Dosis in der kontralateralen Brust berücksichtigt, jedoch nur mit dem halben Wichtungsfaktor für die Mamma.

Für die Bestimmung der Kollektivdosis infolge der Strahlentherapie benötigt man die verschiedenen Zielvolumina, die applizierten mittleren Dosen in den Zielvolumina und die Anzahl der jeweiligen Patienten. Dafür wurden die Angaben der Schweiz beigezogen, welche das Bundesamt für Gesund-

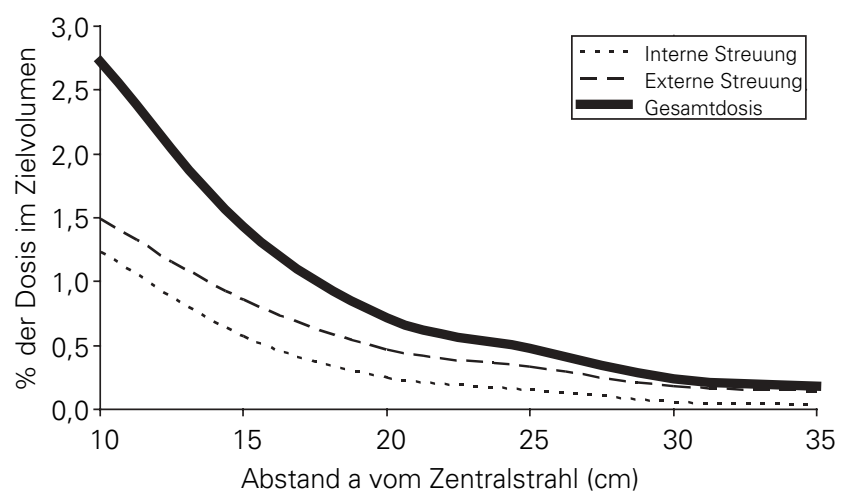

Abbildung 2. Relative Organdosen (bezogen auf die Dosis im Zielvolumen) und ihre Beiträge durch externe und interne Streustrahlung in Abhängigkeit vom Abstand vom Zentralstrahl bei folgenden Parametern: $10 \mathrm{MV} \mathrm{X}$, Feldgröße $10 \mathrm{~cm} \times 10 \mathrm{~cm}$, Isozentrumstiefe $10 \mathrm{~cm}$, Patientendicke $20 \mathrm{~cm}$, Gewebetiefe $10 \mathrm{~cm}$.

Figure 2. Relative organ doses (in relation to the dose in the target volume) and their contribution by external and internal scatter radiation based on the distance to the beam axis at the following parameters: $10 \mathrm{MVX}$, field size $10 \mathrm{~cm} \times 10 \mathrm{~cm}$, depth of the isocenter $10 \mathrm{~cm}$, patient's thickness $20 \mathrm{~cm}$, depth of tissue $10 \mathrm{~cm}$.

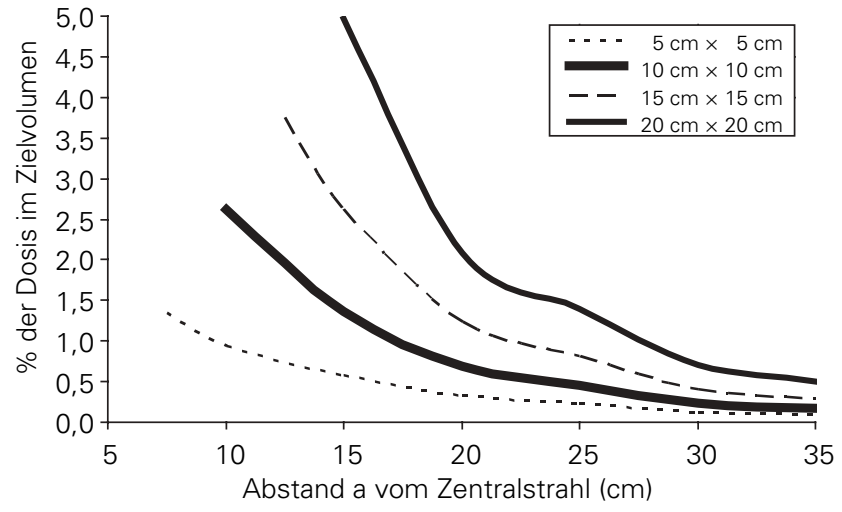

Abbildung 3. Relative Organdosen (bezogen auf die Dosis im Zielvolumen) in Abhängigkeit vom Abstand vom Zentralstrahl für verschiedene Feldgrößen bei folgenden Parametern: $18 \mathrm{MVX}$, Isozentrumstiefe $10 \mathrm{~cm}$, Patientendicke $20 \mathrm{~cm}$, Gewebetiefe $10 \mathrm{~cm}$.

Figure 3. Relative organ doses (in relation to the dose in the target volume) based on the distance to the beam axis for different field sizes at the following parameters: $18 \mathrm{MV} \mathrm{X}$, depth of the isocenter $10 \mathrm{~cm}$, patient's thickness $20 \mathrm{~cm}$, depth of tissue $10 \mathrm{~cm}$.

heit für die Erhebung des nächsten UNSCEAR-Reports ermittelt und weitergeleitet hat [2]. In der vorliegenden Arbeit wurden die palliativen Behandlungen nicht berücksichtigt, da angenommen wird, dass das Risiko einer allfälligen Tumorinduktion nicht manifest wird.

\section{Ergebnisse}

Die Dosen hängen in unterschiedlichem Ausmaß von den Einstellparametern sowie der Lage des Zielvolumens und des Risikoorgans im Patienten ab. Wie in Abbildungen 2 bis 4 gezeigt wird, spielen dabei vor allem die Distanz zwischen Ziel-

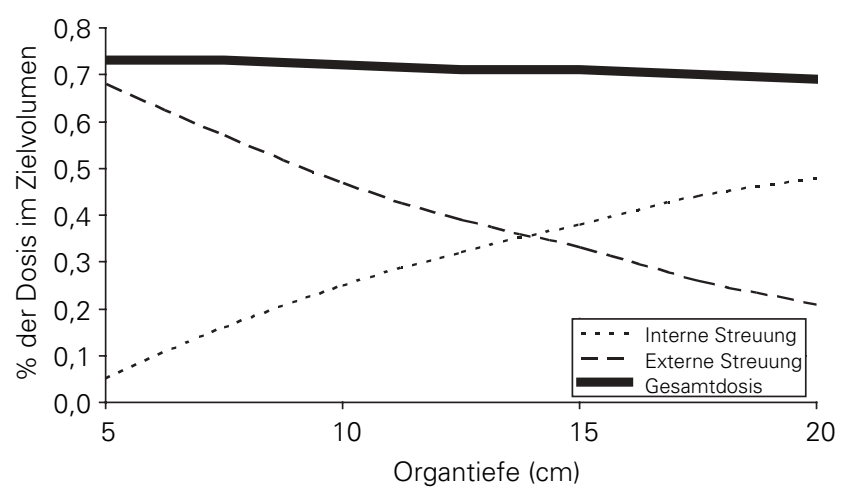

Abbildung 4. Relative Organdosen (bezogen auf die Dosis im Zielvolumen) und ihre Beiträge durch externe und interne Streustrahlung in Abhängigkeit von der Gewebetiefe bei folgenden Parametern: $10 \mathrm{MVX}$, Feldgröße $10 \mathrm{~cm} \times 10 \mathrm{~cm}, 20 \mathrm{~cm}$ Abstand vom Zentralstrahl, Isozentrumstiefe $10 \mathrm{~cm}$, Patientendicke $20 \mathrm{~cm}$.

Figure 4. Relative organ doses (in relation to the dose in the target volume) and their contribution by external and internal scatter radiation in relation to the tissue's depth at the following parameters: $10 \mathrm{MVX}$, field size $10 \mathrm{~cm} \times 10 \mathrm{~cm}, 20 \mathrm{~cm}$ distance from the beam axis, depth of the isocenter $10 \mathrm{~cm}$, patient's thickness $20 \mathrm{~cm}$. 
volumen und Risikoorgan sowie die Feldgröße eine wichtige Rolle. Je größer das Bestrahlungsfeld, desto größer ist der Anteil der internen im Vergleich zur externen Streustrahlung. In Gewebetiefen bis etwa $12 \mathrm{~cm}$ überwiegt der externe Streuanteil ab einer Distanz von 10-20 cm vom Zielvolumen je nach Feldgröße. In größeren Tiefen bleibt der interne Streustrahlenanteil auch in größeren Abständen vom Zielvolumen dominant.

Für klinische Beispiele sind Organdosen und effektive Dosen in Tabelle 1 aufgeführt. Die höchsten Organdosen wurden für die Hoden bei der Rektumbestrahlung (3,2 Sv) sowie für die Hoden und das Colon descendens (je 2,3 Sv) bei der Prostatabestrahlung bestimmt.
Die berechneten effektiven Dosen liegen im Bereich zwischen 60 (Mamma, 50 Gy im Zielvolumen) und 902 mSv (Rektum, 45 Gy, Mann). Mit dem Risiko von 5\% pro Sv für strahleninduzierte Krebstodesfälle beträgt das gesamte Strahlenrisiko für eine Strahlentherapie somit zwischen 0,3\% und 4,5\%. Die effektive Dosis wird als proportional zur Dosis im entsprechenden Zielvolumen angenommen und kann damit auf einfache Weise angepasst werden.

Wie die Berechnungen gezeigt haben, sind die Organdosen außerhalb des Zielvolumens für die meisten Risikoorgane $<1 \%$ der Dosis im Zielvolumen. In wenigen Organen beträgt die Dosis bis etwa 3\%, und für die Hoden bei der Rektumbestrahlung wurden $6,3 \%$ bestimmt. Die drei Organe mit den

Tabelle 1. Mittlere Organdosen und daraus berechnete effektive Dosen für einige Bestrahlungsbeispiele. Werte in mSv. (ZV) bezeichnet ein Organ im oder angrenzend an das Zielvolumen.

Table 1. Average organ doses and resulting effective doses for some examples of radiotherapy. Values in mSv. (ZV) qualifies an organ in or next to the target volume.

\begin{tabular}{|c|c|c|c|c|c|c|c|c|}
\hline Zielvolumen & & Ganzhirn & Larynx & Mamma & $\begin{array}{l}\text { Media- } \\
\text { stinum }\end{array}$ & Rektum & Uterus & Prostata \\
\hline Anzahl Felder & & 2 & 3 & 2 & 3 & 4 & 4 & 4 \\
\hline Strahlenenergie & & $6 \mathrm{MVX}$ & $6 \mathrm{MVX}$ & $6 \mathrm{MV} X$ & $18 \mathrm{MV} \mathrm{X}$ & $18 \mathrm{MV} X$ & $18 \mathrm{MV} X$ & $18 \mathrm{MV} X$ \\
\hline Dosis im ICRU-Punkt (Gy) & & 30 & 74,4 & 50,4 & 54 & 45 & 50,4 & 74 \\
\hline Risikoorgane & $\mathrm{w}_{\mathrm{T}}$ & & & & & & & \\
\hline Ovarien & 0,20 & 12 & 47 & 25 & 54 & $(\mathrm{ZV})$ & $(\mathrm{ZV})$ & \\
\hline Hoden & & 10 & 40 & & 36 & 3220 & & 2287 \\
\hline Rotes Knochenmark & 0,12 & 164 & 687 & 49 & 505 & 206 & 238 & 90 \\
\hline Colon descendens & 0,12 & 15 & 57 & 34 & 64 & $(\mathrm{ZV})$ & $(Z V)$ & 2310 \\
\hline Lungen & 0,12 & 342 & 1446 & 101 & 1541 & 124 & 386 & 79 \\
\hline Magen & 0,12 & 40 & 173 & 78 & 257 & 929 & 804 & 369 \\
\hline Blase & 0,05 & 20 & 52 & 23 & 43 & $(Z V)$ & 20 & $(Z V)$ \\
\hline Brust & 0,05 & 77 & 402 & 71 & 726 & 293 & 195 & \\
\hline Leber & 0,05 & 50 & 186 & 101 & 357 & 1066 & 961 & 453 \\
\hline Ösophagus & 0,05 & 111 & 542 & 102 & 1330 & 260 & 178 & 131 \\
\hline Schilddrüse & 0,05 & 1050 & $(Z V)$ & 70 & 1270 & 65 & 41 & 55 \\
\hline Haut & 0,01 & 52 & 127 & 134 & 119 & 98 & 104 & 148 \\
\hline Knochenoberfläche & 0,01 & 164 & 687 & 49 & 505 & 206 & 238 & 90 \\
\hline \multicolumn{9}{|l|}{ Restorgane (Remainder) } \\
\hline Nebennieren & & 59 & 228 & 126 & 420 & 570 & 420 & 216 \\
\hline Hirn & & $(Z V)$ & 1354 & 19 & 311 & 46 & 34 & 49 \\
\hline Colon ascendens/transversum & & 20 & 70 & 50 & 68 & $(\mathrm{ZV})$ & 1259 & 1910 \\
\hline Dünndarm & & 21 & 78 & 59 & 81 & $(Z V)$ & 1381 & 1492 \\
\hline Nieren & ஸ̊ & 24 & 84 & 20 & 100 & 1488 & 1109 & 1314 \\
\hline Pankreas & O & 47 & 211 & 93 & 328 & 752 & 558 & 249 \\
\hline Milz & 3 & 59 & 238 & 132 & 459 & 570 & 420 & 213 \\
\hline Thymus & $\tilde{W}$ & 495 & 2042 & 84 & 1390 & 94 & 62 & 63 \\
\hline Uterus & & 20 & 50 & 20 & 47 & $(Z \mathrm{~V})$ & $(Z V)$ & \\
\hline \multirow[t]{3}{*}{ Effektive Dosis (mSv) } & Frau & 144 & 401 & 60 & 516 & 273 & 292 & \\
\hline & Mann & 140 & 379 & & 482 & 902 & & 885 \\
\hline & Mittelwert & 142 & 390 & & 499 & 588 & & \\
\hline \multirow[t]{3}{*}{ Effektive Dosis ohne Gonadendosen (mSv) } & Frau & 142 & 392 & 55 & 506 & 273 & 292 & \\
\hline & Mann & 138 & 371 & & 475 & 258 & & 428 \\
\hline & Mittelwert & 140 & 381 & & 490 & 266 & & \\
\hline
\end{tabular}


jeweils höchsten Dosen, bezogen auf die Zielvolumendosis, sind für die in Tabelle 1 aufgeführten klinischen Beispiele in Tabelle 2 zusammengestellt.

Die Häufigkeiten der bestrahlten Zielvolumina in der Schweiz sind in Tabelle 3 aufgeführt [2]. Im Jahre 2003 wurden

Tabelle 2. Die drei Organe mit den jeweils höchsten Dosen bei der Bestrahlung verschiedener Zielvolumina.

Table 2. The three organs with the respective highest dose during the irradiation of different target volumes.

\begin{tabular}{lll}
\hline Zielvolumen & Organ & $\begin{array}{l}\text { Organdosis in \% der Dosis } \\
\text { im Zielvolumen }\end{array}$ \\
\hline Ganzhirn & Schilddrüse & 3,5 \\
& Thymus & 1,7 \\
Larynx & Lungen & 1,1 \\
& Thymus & 2,7 \\
Mediastinum & Lungen & 1,9 \\
& Hirn & 1,8 \\
Mamma & Lungen & 2,9 \\
& Thymus & 2,6 \\
& Ösophagus & 2,5 \\
Rektum & Milz & 0,3 \\
& Nebennieren & 0,3 \\
& Ösophagus & 0,2 \\
Uterus & Hoden & 6,3 \\
& Nieren & 3,3 \\
& Leber & 2,4 \\
& Dünndarm & 2,7 \\
& Colon ascendens/ & 2,5 \\
& transversum & \\
& Nieren & 2,2 \\
& Colon descendes & 3,1 \\
& Hoden & 3,1 \\
& Colon ascendens/ & 2,6 \\
& transversum & \\
\hline
\end{tabular}

Tabelle 3. Häufigste Tumorlokalisationen und ihre Anteile in der Radioonkologie in der Schweiz (nach [2]).

Table 3. Most frequent tumor localizations and their quota in radiooncology in Switzerland (after [2]).

\begin{tabular}{lc}
\hline Zielvolumen & Anteil (\%) \\
\hline Mamma & 21,7 \\
Lunge und Thorax & 6,9 \\
Prostata & 6,7 \\
Kopf/Hals & 5,3 \\
Gynäkologische Tumoren & 4,2 \\
Kolon, Rektum & 4,1 \\
Gehirn & 3,3 \\
Lymphom & 2,9 \\
Gutartige Erkrankungen & 5,8 \\
Palliative Behandlungen & 22,5 \\
Übrige & 16,5 \\
Total & 100,0 \\
\hline
\end{tabular}

14500 perkutane Bestrahlungen in 21 radioonkologischen Zentren mit 44 Linearbeschleunigern und drei Bestrahlungseinheiten $\left({ }^{60} \mathrm{Co}\right)$ durchgeführt. Dies ergibt zwei Bestrahlungen pro 1000 Einwohner. Die Anzahl von weiblichen und männlichen Patienten ist ungefähr gleich [2]. Mit den effektiven Dosen und der Anzahl der Bestrahlungen für die einzelnen Zielvolumina lässt sich die Kollektivdosis aller Strahlentherapien im Hochvoltbereich in der Schweiz damit auf 4255 Personen-Sv berechnen. Dabei sind Bestrahlungen mit palliativer Zielsetzung, mit Elektronenstrahlen sowie die stereotaktischen Bestrahlungen und Ganzkörperbestrahlungen wie auch die Brachytherapien und konventionellen Röntgentherapien nicht berücksichtigt.

\section{Diskussion}

Die Bestimmung von Organdosen außerhalb des Zielvolumens und der daraus berechneten effektiven Dosen in der Radioonkologie ergibt erwartungsgemäß relativ hohe Werte im Vergleich zur Röntgendiagnostik oder Nuklearmedizin. So kann das Risiko für Krebsinduktionen aufgrund einer Strahlentherapie abgeschätzt werden, in Übereinstimmung mit UNSCEAR [13]. Im NRPB-Bericht [8] wird angegeben, dass die Dosis von der Streustrahlung außerhalb des Zielvolumens ein wichtiger Faktor für die Krebsinduktion in der Radiotherapie sei. Im Vergleich zu früher sind heute Krebsbehandlungen in vielen Fällen erfolgreicher, und das Leben von Krebspatienten wird verlängert. Damit hat auch das Strahlenrisiko einer Krebsinduktion für das verlängerte Überleben an Bedeutung zugenommen [10].

Die Dosis in einem Organ wird vor allem von den folgenden Parametern bestimmt: Abstand vom Rand des Zielvolumens und Feldgröße. Sowohl die Strahlenenergie wie auch die Fokus-Oberflächen-Distanz haben keinen großen Einfluss auf die Streustrahlung, bezogen auf die Zielvolumendosis. Mit zunehmender Distanz vom Feldrand nimmt der Beitrag der internen Streustrahlung ab. In größerem Abstand wird die Streustrahlung weitgehend vom Blendensystem bestimmt. Obwohl sich die Beiträge der internen und externen Streustrahlung mit der Gewebetiefe stark ändern, bleibt deren Summe (Gesamtdosis) etwa konstant, wie am Beispiel in Abbildung 4 ersichtlich ist. Die in der Praxis verwendeten Bestrahlungsplanungssysteme unterschätzen meist die Dosen bereits ab einigen Zentimetern vom Feldrand um 25-75\%.

Die Berechnungen nach PERIDOSE wurden für einige Beispiele mit eigenen Messungen nachgeprüft. Es wurden vor allem in größeren Abständen vom Zielvolumen Abweichungen bis maximal $40 \%$ festgestellt. Für die Uterusdosis bei einer Mammabestrahlung (50,4 Gy im Zielvolumen, 6 MV X, Zwei-Felder-Technik, mit Keilfilter, $32 \mathrm{~cm}$ Abstand vom Feldrand) wurden in einem Alderson-Phantom beispielsweise $25 \mathrm{mSv}$ für die gesamte Strahlentherapie bestimmt. Die Berechnung ergab $20 \mathrm{mSv}$. Im Durchschnitt beträgt das Verhältnis zwischen gemessenen und berechneten Organdosen 0,92.

Eine hohe Gonadendosis trägt wesentlich zur effektiven Dosis bei, aufgrund des hohen Wichtungsfaktors von 0,20. 
Dies ist vorwiegend bei Bestrahlungen im Beckenbereich der Fall. Ein Großteil der Patientinnen wird jedoch postmenopausal bestrahlt. Da 92\% der Patienten bei Bestrahlungsbeginn > 40 Jahre alt sind [2], werden die Auswirkungen der Gonadendosen stark überbewertet. Berücksichtigt man die Gonadendosen nicht, so werden die effektiven Dosen stark reduziert. Dies betrifft vor allem Bestrahlungen der Prostata, des Uterus und des Rektums. In Tabelle 1 wurden deshalb auch die effektiven Dosen ohne Berücksichtigung der Gonadendosen angegeben.

Gemäß Jahresstatistiken erfolgen etwa 3\% aller Bestrahlungen mit Elektronen. Bei Elektronenstrahlung liegen die Organdosen etwa um einen Faktor 4 tiefer als bei Photonenstrahlung [14], da der Beitrag der internen Streustrahlung bei Elektronenstrahlung klein ist.

In Abhängigkeit von der Dosis am Entstehungsort und den behandlungsbezogenen Einflussfaktoren wurde in einer Studie die Entstehung von Zweittumoren nach Strahlentherapie mit Röntgenstrahlung und ${ }^{60} \mathrm{Co}-\gamma$-Strahlung untersucht [6]. Danach findet sich eine signifikante Anzahl von Sekundärtumoren in dem mit $\leq 6$ Gy belasteten Volumen. In einer prospektiven Studie soll eine Korrelation zwischen Organdosen und der Inzidenz akuter und chronischer Nebenwirkungen nach Strahlentherapie bei Kindern und Jugendlichen ermöglicht werden [3].

Für ausgewählte Bestrahlungen sind vereinzelt effektive Dosen publiziert worden, so z.B. für die Risikoabschätzung bei der Radiotherapie der Graves-Orbitopathie (effektive Dosis 66,8 mSv) [1]. Die von UNSCEAR [12] publizierten effektiven Dosen für vier Zielvolumina (Hals, Thorax, Pankreas und Gallenblase, Becken) liegen im Bereich zwischen 57 und $1790 \mathrm{mSv}$ (bezogen auf $60 \mathrm{~Gy}$ im Zielvolumen) und sind teilweise wesentlich höher als die Werte in der vorliegenden Arbeit. Allerdings beziehen sich die publizierten Werte auf ${ }^{60}$ Co-Bestrahlungen in den Jahren 1978-1979.

Die Methode der effektiven Dosis wird jedoch auch als ungeeignet für die Abschätzung des Risikos einer Krebsinduktion durch die Strahlentherapie bezeichnet [11]. Dieses Risiko für die kritischen Organe sollte danach allein auf der Basis epidemiologischer Daten von Patienten und nicht mit den von der ICRP [7] für den Strahlenschutz ermittelten Risikofaktoren abgeschätzt werden. Allerdings wird eingeräumt, dass bei kleinen Dosen infolge Streustrahlung das Konzept der effektiven Dosis nützlich sein könnte.

Aus der Studie von Aroua et al. [2] geht hervor, dass je nach Zielvolumen zwischen $67 \%$ und $100 \%$ der Patienten bei Bestrahlungsbeginn > 40 Jahre alt sind, im Mittel 92\% der Patienten. Das stochastische Strahlenrisiko beträgt gemäß ICRP [7] $5 \%$ pro Sv, gemittelt über die gesamte Bevölkerung. Nach dem multiplikativen Modell der ICRP ist das Strahlenrisiko im Alter $>40$ Jahre zum Zeitpunkt der Exposition jedoch $<2,5 \%$ pro Sv und fällt kontinuierlich auf 0 im Alter von 90 Jahren ab. Das Strahlenrisiko des Patientenkollektivs wird somit um mindestens einen Faktor 2 reduziert. Berücksichtigt man die Gonadendosen nicht (vgl. Tabelle 1), so beträgt die Kollektivdosis 3211 Personen-Sv.

Spezielle Fragestellungen betreffen die Bestrahlungen mit Elektronen, die Brachytherapie, die nuklearmedizinische Therapie, die konventionelle Röntgentherapie sowie die ophthalmologischen und intrakoronaren Bestrahlungen. Diese müssen gesondert betrachtet werden.

\section{Danksagung}

Für die Zurverfügungstellung des Programms PERIDOSE mit den notwendigen Hinweisen bedanken wir uns bestens bei P.-H. van der Giessen.

\section{Literatur}

1. Akmansu M, Dirican B, Bora $\mathrm{H}$, et al. The risk of radiation-induced carcinogenesis after external beam radiotherapy of Graves' orbitopathy. Ophthalmic Res 2003;36:150-3.

2. Aroua A, Moeckli R, Valley JF, et al. Enquête sur la pratique da la radiothérapie en Suisse en 2003. Rapport. Lausanne: Institut Universitaire de Radiophysique Appliquée, 2006.

3. Bölling T, Schuck A, Hesselmann S, et al. Behandlungsassoziierte Spätfolgen nach Strahlentherapie maligner Erkrankungen im Kindes- und Jugendalter. Strahlenther Onkol 2006;182:443-9.

4. Diallo I, Lamon A, Shamsaldin A, et al. Estimation of the radiation dose delivered to any point outside the target volume per patient treated with external beam radiotherapy. Radiother Oncol 1996;38:269-71.

5. Dörr W, Bertmann S, Herrmann T. Radiation-induced lung reactions in breast cancer therapy. Strahlenther Onkol 2005;181:567-73.

6. Dörr W, Herrmann T. Second primary tumors after radiotherapy for malignancies. Strahlenther Onkol 2002;178:357-62.

7. International Commission on Radiological Protection. 1990 recommendations of the International Commission on Radiological Protection. ICRP Publication 60. 0xford: Pergamon Press, 1991.

8. National Radiological Protection Board. Risks of second cancer in therapeutically irradiated populations. Report of an advisory group on ionising radiation. Doc NRPB 2000;11:11-5.

9. Roth J, Roser HW, Schnekenburger B. Gedanken zur effektiven Dosis in der Radio-Onkologie. In: Welker K, Zink K, Hrsg. Medizinische Physik 2001. Berlin: DGMP, 2001:307-8.

10. Schneider U, Lomax A, Pemler P, et al. The impact of IMRT and proton radiotherapy on secondary cancer incidence. Strahlenther Onkol 2006; 182:647-52.

11. Trott KR, Kamprad F. Estimation of cancer risks from radiotherapy of benign diseases. Strahlenther Onkol 2006;182:431-6.

12. UNSCEAR 1993 Report. Sources and effects of ionizing radiation. New York: United Nations, 1993.

13. UNSCEAR 2000 Report. Sources and effects of ionizing radiation, vol I: Sources, Annex D. New York: United Nations, 2002.

14. Van der Giessen PH. Peridose, a software program to calculate the dose outside the primary beam in radiation therapy. Radiother Oncol 2001; 58:209-13.

15. Williams G, Zankl M, Drexler G. The calculation of dose from external photon exposures using reference human phantoms and Monte Carlo methods. Part IV: Organ doses in radiotherapy. GSF-Bericht S-1054. München: GSF, 1987.

\author{
Korrespondenzanschrift \\ Prof. Dr. Jakob Roth \\ Radiologische Physik \\ Radio-Onkologie \\ Universitätsspital \\ 4031 Basel \\ Schweiz \\ Telefon (+41/61) 265-3141, Fax -3135 \\ E-Mail:jroth@uhbs.ch
}

